\title{
Re-Ingeniería de apatita natural para soporte de tejidos óseos
}

\author{
Re-Engineering of Natural Apatite for Scaffold of \\ Hard Tissues
}

\author{
Héctor Pesenti ${ }^{1,2}$, Rolando Ríos ${ }^{2}$, Matteo Leoni ${ }^{1}$, \\ Antonella Motta ${ }^{1}$, Paolo Scardi ${ }^{1}$
}

\footnotetext{
${ }^{1}$ Dipartimento di Materiali, Univers ità di Trento, via Mesiano, 7738123 Trento, Italia.

${ }^{2}$ Instituto de Materiales y Procesos Termomecánicos, Universidad Austral de Chile, General Lagos 2086, Chile. e-mail: pesentih@gmail.com
}

\begin{abstract}
RESUMEN
Actualmente los grandes progresos en la medicina ortopédica, traumatológica y odontológica, han originado una gran necesidad por biomateriales, especialmente por aquellos productos que tienen una composición en base de ortofosfato de calcio, el cual es similar a la estructura inorgánica de los huesos. Ante este escenario, se ha generado una intensa búsqueda de nuevos orígenes y recursos, siendo los materiales naturales un foco de importantes estudios, debido a que estos pueden abastecer plenamente la gran demanda por biomateriales. Los estudios de estos materiales naturales han reportado interesantes avances, especialmente en lo que respecta a la regeneración de tejidos biológicos en medicina reparativa. De manera de seguir contribuyendo con este desafío, se analizaron muestras de minerales provenientes de un extenso depósito geológico hidrotermal, para proponer estos materiales como posible fuente de recursos. Los minerales extraídos fueron disgregados, para posteriormente fabricar probetas cilíndricas mediante Sinterización por Arco de Plasma, para luego ser biológicamente testeadas. La caracterización mineralógica del material extraído, reveló altos contenidos de fosfatos en forma de apatita, seguidas de pequeñas cantidades de otras especies mineralógicas asociadas, como el cuarzo y yeso. Dadas estas características, el polvo de mineral presentó una buena disposición a la sinterización, siendo capaz de densificar completamente a temperatura sobre $\operatorname{los} 1000^{\circ} \mathrm{C}$. Los resultados de la sinterización fueron correlacionados con las fases obtenidas con las diferentes temperaturas de proceso, presentando la apatita muy buena estabilidad termoquímica. Pruebas preliminares in vitro de proliferación y adhesión celular de osteoblastos humanos, MG63, en las muestras preparadas, parecen ser bastantes prometedoras, siendo la probeta sinterizada a $1200^{\circ} \mathrm{C}$ la que presentó la mejor activación celular, semejante al comportamiento biológico de las hidroxiapatitas comerciales reportadas.
\end{abstract}

Palabras clave: Depósito Hidrotermal, Hidroxiapatita Natural, Sinterización por arco plasma, Biocompatibilidad.

\section{ABSTRACT}

Recent progresses in orthopedic medicine, traumatology and odontology have created a great requirement for biomaterials, especially those products having a composition based on calcium orthophosphate, which is similar to inorganic bone structure. Given this situation, there has been an intense search for new origins and resources of biomaterials. The natural occurring materials have been a focus of important studies because they can satisfy the great demand of biomaterials. Studies on these natural materials have reported interesting progresses in relation to the biological tissue regeneration for reparative medicine. In this research work, samples of minerals of a geological hydrothermal deposit were analyzed and this material has been proposed as an important resource of biomaterials. The minerals extracted were disaggregated, transformed into cylindrical specimens by Arc Plasma Sintering, and tested for biological compatibility. The mineralogy of the extracted material revealed a high content of phosphates in the form of apatite, followed by small amounts of other associated mineralogical species such as quartz and gypsum. Under these characteristics, the mineral powder showed a good disposition to sintering; being able to densify at temperatures above $1000^{\circ} \mathrm{C}$. The 
results of sintering were correlated with the different phase evolutions with the process temperatures; with apatite showing very good thermochemistry stability. Preliminary, biological "in vitro" tests of cells attachment and proliferation of human osteoblasts, MG63, indicated that, the prepared specimen sintered at $1200^{\circ} \mathrm{C}$ appear to be quite promising, as it showed the best cell activation similar to the biological behavior of the reported commercial hydroxyapatite.

Keywords: Hydrothermal Deposit, Natural Hydroxylapatite, SPS, Biocompatibility.

\section{INTRODUCCIÓN}

La declinación fisiológica en los tejidos duros, en casos de tumores o infecciones o traumas, son los mayores problemas en cirugía ortopédica y maxifaciales [1]. Actualmente, tales afecciones son reparadas mediante una gran variedad de bioderivados o materiales sintéticos. Sin embargo, estos materiales deben ser mecánica y químicamente estables, o estar limitados a no inducir efectos citotóxicos o generar alguna reacción biológica negativa. Asimismo, estos deben ser capaces de promover la formación de nuevos tejidos y estimular la osteointegración, lo que genera una mejor interacción del entorno biológico con la superficie del implante [2] sin causar un rechazo inmunológico grave.

Los actuales avances en la medicina reparativa, han generado una gran demanda por biomateriales, especialmente, por aquellos productos que tienen una composición a base de ortofosfato de calcio, similar a la forma cristaloquímica de la estructura inorgánica de los huesos. Este gran suceso es por las compatibles propiedades biológicas y por la total ausencia de toxicidad de sus constituyentes. Esto evidencia la amplia gama de materiales de $\mathrm{Ca}_{10}\left(\mathrm{PO}_{4}\right)_{6}(\mathrm{OH})_{2}$ fabricados y usados actualmente en medicina. Estos productos cerámicos destinados a sustentar las necesidades biomédicas, están elaborados de manera que sean reabsorbibles o no-reabsorbibles, de modo compacto o poroso, dirigidos para el revestimiento de prótesis, como para biocementos, cementos inyectables, materiales compuestos, portadores de droga, y así como tantas otras formas de uso.

Los ortofosfatos, especialmente aquellos de calcio, pertenecen una amplia familia de avanzados compuestos químicos denominados mineralógicamente como apatitas, siendo la hidroxiapatita, $\mathrm{Ca}_{5}\left(\mathrm{PO}_{4}\right)_{3}(\mathrm{OH})$, una de las formas más representativas y más usadas en la medicina reparativa [ $\underline{3}, \underline{4}]$. Normalmente, las apatitas pueden ser conseguidas mediante síntesis química, o bien pueden encontrarse en forma natural sobre la corteza terrestre gracias a procesos biológicos, o por una eventual alteración geológica.

En cuanto a la posibilidad de obtener las apatitas por vía química, existen factores claves que restringen normalmente su producción tales como: la limitada facilidad de obtención, baja disponibilidad, moderada homogeneidad, complejidad en los procesos y equipos utilizados [5]. En cambio los biomateriales de origen natural, han comenzado a irrumpir en importancia gracias a los buenos resultados en aplicaciones médicas, y a las excelentes respuestas biológicas en comparación a los tradicionales materiales sintéticos.

Esta fascinación por el estudio de materiales naturales se debe a que muchos de los exoesqueletos de animales (génesis biológica), y otras fuentes de origen natural (génesis geológicas), proveen una cuantiosa fuente de recursos de materiales biocompatibles [묵] .

En este contexto, estructuras perfectamente mineralizadas de animales marinos, tales como erizos de mar, esponjas naturales, moluscos cefalópodos, algas rojas mineralizadas, coral, entre otros, son modelos ideales de soportes para la regeneración de tejidos óseos [6]. Esto se debe a las interesantes ventajas que ofrecen en la configuración de la microestructura, siendo similar a los huesos, así como también dispone de una alta macroporosidad para la vascularización, la proliferación de células óseas, y la angiogénesis [8]. Dadas las consideraciones anteriores, los materiales de origen biológico, han tenido un gran interés médico, especialmente por los buenos resultados en sus aplicaciones [6].

Sin embargo, a pesar de los grandes esfuerzos fisicoquímicos realizados sobre la superficie de estos andamios, aun presentan algunas dificultades, tales como las altas velocidades de disolución, la reducida durabilidad y limitada estabilidad [9]. Asimismo, han presentado algunos casos de intolerancia biológica [10], lo que ha causado un arduo estudio para incrementar el rendimiento de estos biocerámicos naturales.

Por otra parte, las apatitas de origen geológico son minerales accesorios en casi todas las rocas ígneas, así como también constituyente de varias rocas sedimentarias y metamórficas. Las más comunes variedades de especies mineralógicas están representadas por la serie isomorfa con fórmula química general $(\mathrm{Ca}, \mathrm{Na}$, $\mathrm{Mg})_{10-\mathrm{x}}\left(\mathrm{PO}_{4}\right)_{6-\mathrm{x}}\left(\mathrm{CO}_{3}\right)_{\mathrm{x}}(\mathrm{OH}, \mathrm{F}, \mathrm{Cl})_{2-\mathrm{x}-2 \mathrm{y}}\left(\mathrm{CO}_{3}\right)_{\mathrm{y}}$, de las cuales las especies más comunes son la Fluoroapatita, $\mathrm{Ca}_{5}\left(\mathrm{PO}_{4}\right)_{3} \mathrm{~F}$, Clorapatita, $\mathrm{Ca}_{5}\left(\mathrm{PO}_{4}\right)_{3} \mathrm{Cl}$, Francolita $\mathrm{Ca}_{10}\left(\mathrm{PO}_{4}\right)_{6-\mathrm{x}}\left(\mathrm{CO}_{3} \mathrm{~F}\right)_{\mathrm{x}}(\mathrm{OH}, \mathrm{F}, \mathrm{Cl})_{2}$, y la Hidroxilapatita, $\mathrm{Ca}_{5}\left(\mathrm{PO}_{4}\right)_{3} \mathrm{OH}[12,13]$. Estas pueden estar presentes en depósitos, que presentan altas concentraciones de ortofosfatos y con una interesante homogeneidad, como son los yacimientos de guano o fosforita o también en 
ocasionales depósitos hidrotermales, que podrían abastecer la creciente demanda de bioderivados para la elaboración de cerámicos en aplicaciones médicas.

En cuanto a distinguir las distintas variedades criptocristalinas de la apatita, no son muy fáciles de reconocer por los métodos instrumentales convencionales, por lo cual, generalmente es necesario complementar su estudio con otros sofisticados métodos de análisis. No obstante, previos estudios desde depósitos fosfáticos sedimentarios han demostrado una favorable biocompatibilidad.

Según lo reportado, un material siliclastico con altos contenido de francolita provenientes de huesos fósiles y precipitados marinos denominado fosforita, al densificarse térmicamente, forma una matriz vidriosa que presenta un soporte con atrayentes propiedades bioactivas, semejante a los biovidrios-cerámicos comercialmente disponible [7].

Atendiendo la necesidad de buscar nuevos recursos para la creciente necesidad de productos para Biocomponentes, se ha considerado estudiar, desde el punto de vista de la re-ingeniería de los materiales, el potencial de los depósitos geológicos de origen hidrotermal con altas concentraciones y homogeneidad de fosfatos. Siendo el candidato, un mineral fosfático extraído de un gran depocentro del norte de Chile. La investigación de estos cerámicos naturales, se basa en la caracterización cristaloquímica y consolidación de polvo mediante sinterización de plasma arco para generar los preliminares análisis de biocompatibilidad.

\section{MATERIALES Y MÉTODOS}

\subsection{Origen del Material Analizado}

Las muestras fueron suministradas por la "Compañía Minera Formas" en la cantidad necesaria para los diferentes análisis desde un yacimiento minero de origen hidrotermal, situado en la zona suroriental de la cordillera de Domeyko en la provincia de Taltal $[14,15]$. Este sector es parte de un arco magmático denominado "La Negra", en el cual su actividad geológica fue vital para la formación de importantes depocentros de variadas mineralogénesis en la zona. Asimismo, esta área se caracterizó por una rica fauna de animales marinos que habitaron la costa norte de Chile durante el Mioceno Medio-Plioceno Tardío (fuente de fosfatos) y por la expansión marina junto a la subsidencia del Rift por la tectónica extensional desde el periodo Jurásico tardío $[\underline{16}, \underline{17}]$.

En relación a estudios mineralógicos, las apatitas de estas zonas normalmente están asociadas a minerales como la actinolita, la magnetita, el yeso, y a alteraciones de albita y mica sericita. Además, estos ortofosfatos cuentan con grupos funcionales de $\mathrm{OH}-, \mathrm{F}-$ and $\mathrm{Cl}-$. De acuerdo a lo reportado cuantitativamente, el depósito en estudio presenta entre un 32 y $36 \%$ de $\mathrm{P}_{2} \mathrm{O}_{5}$ [18-20], que lo hacen ser un interesante candidato para la elaboración de bioderivados.

\subsection{Preparación de Sinterizados}

El material disponible fue finamente disgregado y homogeneizado en un molino "FRISH PULVERISETTE 9 vibrating-Cup" a condiciones normales y a baja energía de molienda.

Posteriormente, los polvos obtenidos fueron densificados mediante Sinterización de Arco Plasma (Spark Plasma Sintering, SPS), en un equipo DR.SINTER 1050 con electrodos de grafito, para generar tres discos compactos en tres set de probetas con diámetros aproximados de $25 \mathrm{~mm}$, y $10 \mathrm{~mm}$ de espesor. Las probetas fueron obtenidas bajo una presión uniaxial de 30MPa durante 5 min en atmósfera de Argón a temperaturas de $1000^{\circ} \mathrm{C}, 1100^{\circ} \mathrm{C}$, y $1200^{\circ} \mathrm{C}$. Los conjuntos de probetas elaboradas para propósitos experimentales fueron nominados S-APAT-1000, S-APAT-1100 y S-APAT-1200 respectivamente.

No fue posible experimentar probetas a menor temperatura debido a la débil cohesión entre las partículas, disgregándose fácilmente al tacto. Sin embargo, sobre $1200^{\circ} \mathrm{C}$, existió una evidente sublimación de las componentes del material, esto fue verificado por la precipitación de compuestos blancos en la zona de evacuación de gases en la máquina de sinterización, lo que causaba obstrucción de las vías.

Posteriormente, todas las probetas densificadas fueron térmicamente tratados a $700^{\circ} \mathrm{C}$ por $2 \mathrm{~h}$, con la intención de remover cualquier residuo de grafito difundido desde los electrodos a la superficie de los sinterizados. Todas las superficies fueron pulidas mediante papel abrasivo de corindón (grano 320, 800, 1200 y 3200 ), y finalmente con pasta de diamante de $5 \mu \mathrm{m}$ para realizar posteriormente los respectivos test biológicos.

\subsection{Caracterización de las probetas}

Para la caracterización de las probetas, patrones de Difracción de Rayos-X fueron obtenidos mediante un 
difractómetro de polvos Bragg-Brentano marca THERMO modelo ARL X'TRA, basado en un goniómetro vertical $\theta-\theta^{\circ}$ con un detector estado sólido (Si-Li) y enfriador termoeléctrico Peltier. Los datos fueron adquiridos con tubo de blanco metálico de $\mathrm{K}_{\alpha}$ Mo en un rango $10^{\circ}-150^{\circ} 2 \theta^{\circ}$ y un tiempo de conteo de 25 seg por cada $0.05^{\circ}$ de paso. Para profundizar en los aspectos químicos, fueron realizados espectros IR en un espectrofotómetro de Infrarrojo de Transformada de Fourier marca Perkin Elmer Spectrum One, que registra un barrido de 4000-650 $\mathrm{cm}^{-1}$ con una resolución de $4 \mathrm{~cm}^{-1}$, que además está equipado con un accesorio de Reflectancia Atenuada Total (ATR). Los registros espectrales de los polvos fueron hechos en modo de reflectancia con punta de diamante/ZnSe y analizados con un software denominado Spectrum v.5.3.0.

Análisis elemental e imágenes fueron realizados por un Microscopio de Barrido Electrónico marca FEI modelo XL30 ESEM, que además se encuentra equipado con un sistema de microanálisis a energía dispersiva EDAX FALCON. Los datos e imágenes fueron obtenidos a $15 \mathrm{keV}$ en modo ESEM, sin metalización y a 0.9 torr de presión de agua.

\subsection{Test In Vitro de Adhesión Celular}

Las series de muestras elaboradas con distintos parámetros térmicos y replicadas en tres, cada una fue desinfectada en etanol al $70 \%$ a $4{ }^{\circ} \mathrm{C}$ durante una noche, y lavada con agua destilada esterilizada con la finalidad de hacer más eficaz el cultivo. Las probetas fueron ubicadas en una placa de cultivo de poliestireno de 24 pocillos planos. Cada uno de los pocillos fueron llenados directamente con una suspensión de osteoblastos humanos de la línea celular MG63 (osteoblastos humanos aislados de osteosarcoma) cultivados en DMEM (Dulbecco's Modified Eagle Medium, solución de 10\% suero fetal bovino, $1 \%$ antibiótico y antimicótico) con una concentración $3 \times 10^{5}$ células $/ \mathrm{ml}$ en Tripsina al $0,1 \%$.

Posteriormente se dispuso los terrenos de cultivos completamente sobre las probetas, se efectuó la incubación a $37^{\circ} \mathrm{C}$ en una atmósfera de aire/ $\mathrm{CO}_{2}$ humidificada al $95 \%$ durante $48 \mathrm{~h}$, con cambio de terreno a las $24 \mathrm{~h}$.

Al final del cultivo, la fracción de células no adheridas a la superficie de las probetas fueron removidas, para inmediatamente ser lavadas en PBS (solución tampón de fosfato, $\mathrm{pH}$ 7.2), y sucesivamente fijadas químicamente a la superficie de cultivo con una solución de glutaraldehido $(2.5 \%$ en $0.1 \mathrm{M}$ de cacodilato de sodio, $\mathrm{pH}$ 7.2) durante $30 \mathrm{~min}$, y sumergidas 3 veces en una solución tampón de cacodilato de sodio $(0.1 \mathrm{M}$, $\mathrm{pH}$ 7.2).

Luego, cada muestra fue deshidratadas en una serie de distintos grados de etanol $(500 \mu \mathrm{L}$ of $30 \%, 50 \%$, $70 \%, 90 \%$, y 2 veces en $100 \%$ etanol) a cada 10 min y secada al aire. Finalmente, cada muestra fue montada a un portaobjeto de aluminio con un adhesivo de carbono, para realizar las respectivas observaciones morfológicas en ESEM a una presión parcial de aproximadamente de 0.9Torr, de tal modo de poder analizar la adhesión celular en términos cuantitativos.

\section{RESULTADOS Y DISCUSION}

\subsection{Caracterización del Mineral}

En tanto los minerales disgregados por acción mecánica, bajo el microscopio óptico, se observaron en ellos una significativa densidad de microcristales blancos y translucientes de similares características morfológicas y de clivaje. Asimismo, pequeños cristales de color rojo y verde se distinguieron pero no en un número significativo, probablemente estos minerales están asociados a la apatita en la compleja génesis hidrotermal del yacimiento [17]. De acuerdo a lo apreciado desde el perfil de difracción del mineral de partida (figura 1), fue posible observar la presencia de dos tipos de fases de semejantes características cristalinas: Hidroxiapatita, $\mathrm{Ca}_{5}\left(\mathrm{PO}_{4}\right)_{3}(\mathrm{OH})_{2}$ (ficha PDF 73-0293), y Hidroxi-cloro-Apatita, $\mathrm{Ca}_{5}\left(\mathrm{PO}_{4}\right)_{3} \mathrm{Cl}_{\mathrm{x}}(\mathrm{OH})_{2-\mathrm{x}}$ (ficha PDF 70-0794), cuáles han sido representadas con la letra $a$. 


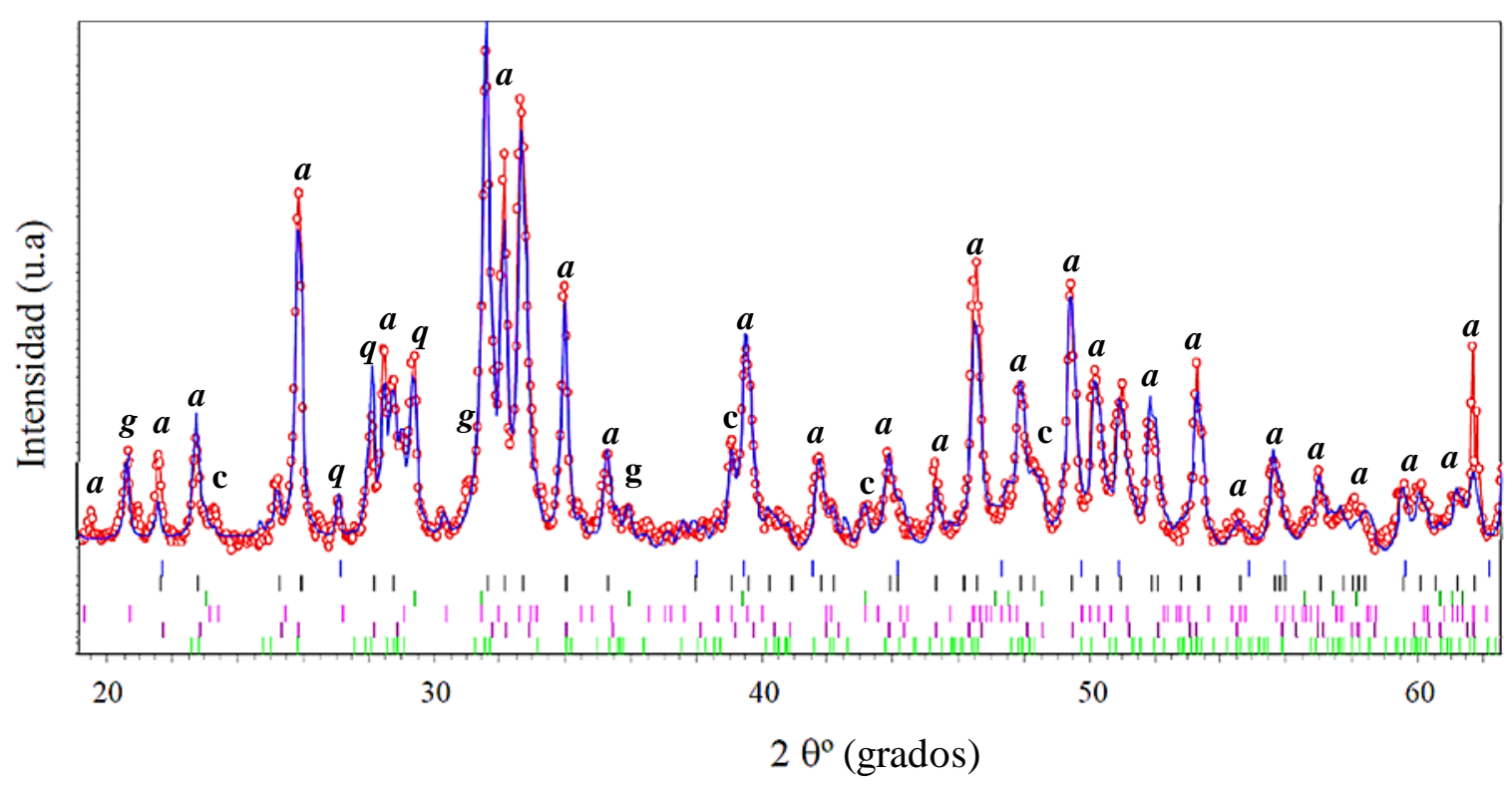

Figura 1: Perfil de difracción del mineral inicial (puntos rojos) y el resultado de refinamiento del perfil mediante el método de Rielveld (línea Azul). Fases reconocidas para el ajuste fueron $(a)$ hidroxiapatita, $(c)$ calcita, $(g)$ yeso, y $(q)$ cuarzo.

Este perfil conduce a la conclusión que existe una posible sustitución iónica ( $\mathrm{Cl}$, F y el carbonato) en la estructura de la apatita, lo que causa una ligera desviación de las posiciones de las intensidades en los valores esperados desde la base de datos de la ICDD para estas estructuras cristalinas, las cuales no pueden ser excluidas. Asimismo, el yeso (CaSO4•2H2O, ficha PDF 33-0311), está presente en una menor fracción; la calcita (CaCO3, ficha PDF 83-0578) también fue claramente identificada. Por otro lado, la alta presión y la baja temperatura de la génesis del depósito posiblemente dieron cobertura a la transformación de alguna fase polimorfa de la sílice. Cuantificando las fases presentes en la muestra inicial desde el análisis del perfil de difracción mediante el método de Rietveld, y usando el software TOPAS v.3.0 (Bruker AXS GmBH, Alemania), fue posible prever un contenido neto de la apatita en la muestra de aproximadamente $87 \%$. Por otra parte, el cuarzo y la coesita fueron incluidas en el afinamiento del patrón de difracción para satisfacer un set de picos que justifican la presencia de la sílice, cuantitativamente este óxido está bajo el 1\% (tabla 1). No obstante, algunas menores fases aún no han sido identificadas, debido a la presencia de la fase apatita que causa un gran predominio grafico en el perfil de difracción afectando la señal de las otras fases menores.

Tabla 1: Balance y Análisis cuantitativo mediante el Método Rietveld y EDS. Porcentajes de las fases y los respectivos elementos químicos: oxígeno y carbón hacen el balance de $100 \%$.

\begin{tabular}{l|l|l|l|l|l|l|l|l|l|l}
\hline & DXR & $\mathrm{P}$ & $\mathrm{Ca}$ & $\mathrm{F}$ & $\mathrm{Cl}$ & $\mathrm{Si}$ & $\mathrm{Na}$ & $\mathrm{K}$ & $\mathrm{Mg}$ & $\mathrm{S}$ \\
\hline Hidroxiapatita & 82,7 & 15,3 & 33 & -- & -- & -- & -- & -- & -- & -- \\
\hline Sílice & 0,4 & -- & -- & -- & -- & 0,2 & -- & -- & -- & -- \\
\hline Calcita & 6,7 & -- & 2,7 & -- & -- & -- & -- & -- & -- & -- \\
\hline Yeso & 5,8 & -- & -- & -- & -- & -- & 1,1 & -- & -- & 1,6 \\
\hline Hidroxicloroapatita & 4,4 & 0,6 & 1,2 & -- & 0,2 & -- & -- & -- & -- & -- \\
\hline DRX & 100 & 15,9 & 36,9 & -- & 0,2 & 0,2 & 1,2 & -- & -- & 1,6 \\
\hline EDS & 100 & 19,4 & 28,6 & 3,2 & 2,1 & 0,8 & 0,9 & 0,3 & 0,5 & 1,8 \\
\hline
\end{tabular}

Los resultados cuantitativos de las fases presentes en la muestra de polvo inicial (APAT-01), pueden ser comparados con un análisis de EDS mediante un balance elemental. De acuerdo a los resultados obtenidos, podría indicarse que ambos métodos se complementan siendo suficientemente bueno. Con respecto a los resultados conseguidos, la proporción $\mathrm{Ca} / \mathrm{P}$ es de aproximadamente $3: 2$, valor equivalente a los valores promedios presentes en los huesos humanos. La baja concentración de otros elementos tales como alcalinos, halógenos y sílice parecen ser sin importancia para los objetivos de estos materiales (tabla 1). No obstante, puede manifestarse algunas diferencias por la baja sensibilidad del EDS a elementos de bajo número atómico 
en comparación a aquellos que presentan un número atómico mayor.

Información adicional química del material de partida fueron obtenidas desde espectrometría FT-IR en absorbancia (figura 2), el espectro muestra una clara presencia de las bandas de vibración características de los grupo funcionales del fosfato en la apatita cerca de $\operatorname{los} 1000 \mathrm{~cm}^{-1}$. La presencia de fases menores identificadas por difracción de rayos-X y elementos livianos adicionales detectado por espectrometría de energía dispersiva, son también notablemente corroborados por IR. De hecho, las bandas del carbonato en el plano de estiramiento, representan el ion en la estructura de la apatita, mientras que las bandas del silicato son observadas cerca los $1150 \mathrm{~cm}^{-1}$ que coincide con el sulfato, lo que resulta la presencia de la sílice y el yeso respectivamente. En los rangos de altas intensidades de absorción, sobre $3000 \mathrm{~cm}^{-1}$, es señal de coordinación de los iones hidroxilo/flúor, que se le atribuye al parcial reemplazo del ion fluoruro por el grupo hidroxilo en la estructura de la apatita [21].

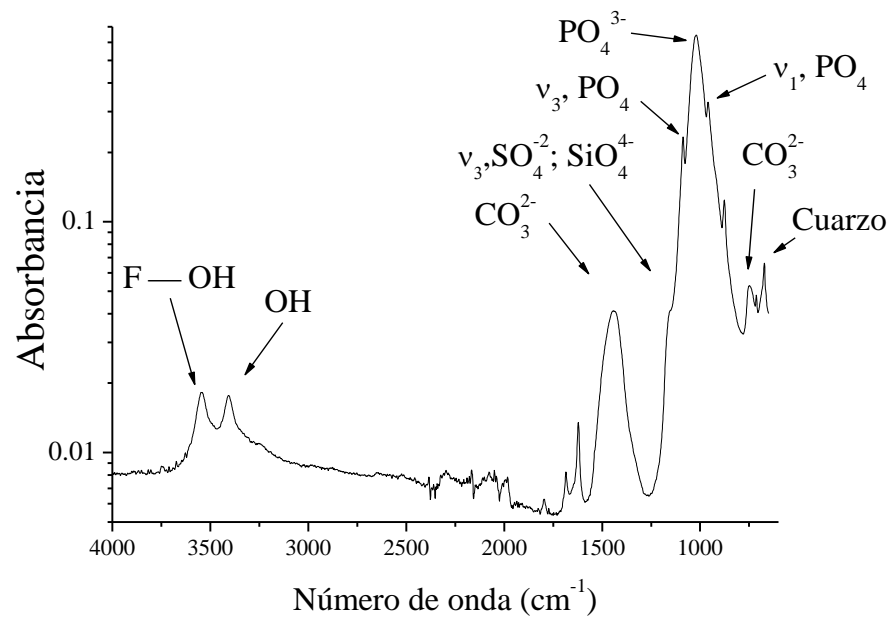

Figura 2: Espectro FT-IR de la apatita hidrotermal, material de partida.

\subsection{Sinterizados de Plasma Arco}

La producción de probetas cilíndricas de apatita hidrotermal mediante arco plasma (APAT-01), fueron realizadas a temperaturas superiores a $1000^{\circ} \mathrm{C}$, dado que a menores temperaturas los polvos no generaron una buena unión entre partículas, permaneciendo escasamente sinterizados. Sin embargo, probetas consolidadas por SPS a $1000^{\circ} \mathrm{C}$ (S-APAT-1000) se observó una significativa cohesión de los polvos, e incluso se evidencia una buena calidad superficial. Pero sin duda, el set sinterizado a $1100^{\circ} \mathrm{C}$ (S-APAT-1100) fue el mejor, exhibiendo una ventajosa resistencia mecánica a las herramientas abrasivas para la preparación superficial.

De igual forma, el perfil de difracción del set S-APAT-1100 se observaron características análogas a aquellas distinguidas en el set de probetas S-APAT-1000. Además se suma que no existe ningún indicio de descomposición de la hidroxiapatita (fase $a$ en figura 3) por presión de compactación o por temperatura, confirmando una buena estabilidad cristaloquímica del polvo cerámico al proceso de sinterización. A mayores temperaturas, el set S-APAT-1200, también evidencia una muy buena consolidación, con ninguna variación cristaloquímica, solo la ligera constitución de la fase flogopita $(f)$, en comparación a las probetas producidas a más baja temperatura. Este último set de probetas presenta una superficie brillante y de un blanco traslucido, indicando que se ha producido una fracción de fase no cristalina o vidriosa (figura 3) que consolida una matriz con una resistencia mecánica muy importante a la presión con herramientas de pulido. Esto también fue reportado por Pesenti et al. [7] en sinterizados de un material natural fosfosiliciclástico. 


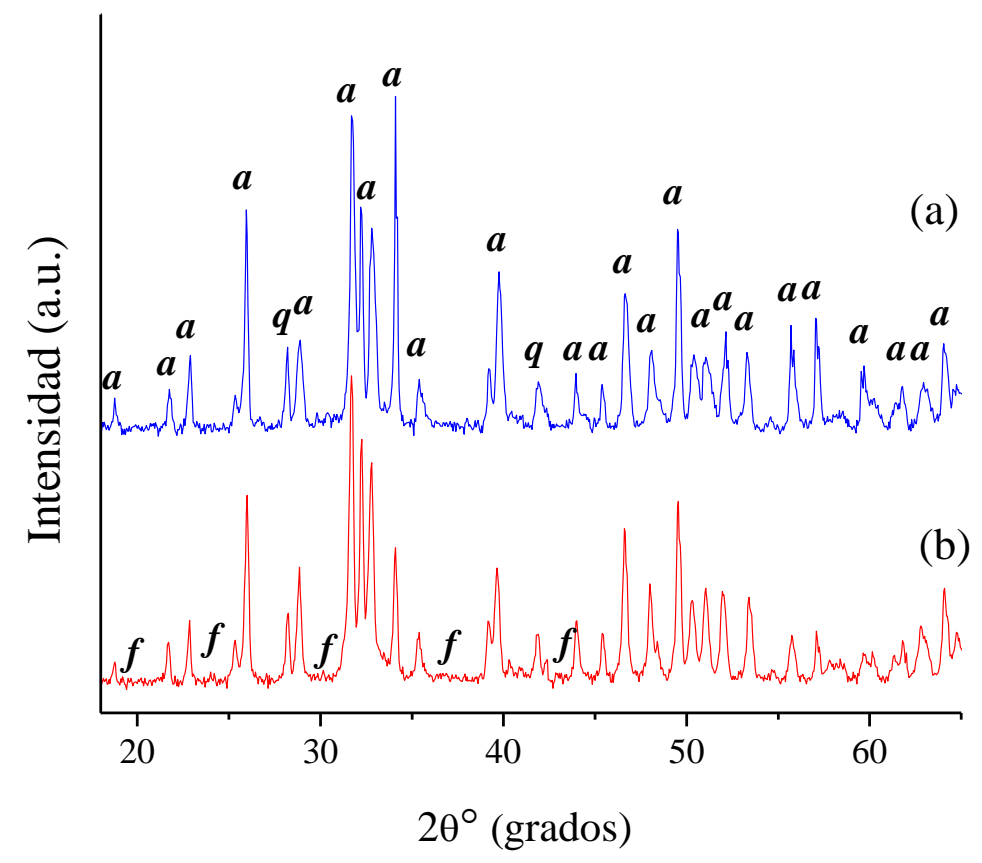

Figura 3: Los perfiles de difracción muestran la evolución de las fases presentes: $a$, hidroxiapatita; $q$, Cuarzo; y $f$, Flogopita, durante el proceso de sinterización de los espécimenes (a) S-APAT-1000 y (b) S-APAT-1200.

Con respecto al análisis de los patrones de difracción, es posible observar la paulatina reducción de las fases distintas a la apatita, formación de la fase no cristalina y la aparición de la flogopita a $1200^{\circ} \mathrm{C}$. Pues es indiscutible además, que exista una gran variación en las intensidades relativas de los picos de la apatita con respecto a los polvos de inicio (APAT-01), que es también evidenciado mediante el refinamiento del perfil de difracción por el método de Rietveld. Sin embargo, se observa una esperada contracción de las reflexiones, indicando un significativo incremento de los dominios cristalinos a causa de los procesos de recristalización a alta temperatura.

Tabla 2: Datos de la composición elemental obtenida mediante EDS de los distintos set de probetas, y valores reportados del hueso y la hidroxiapatita comercial.

\begin{tabular}{c|c|c|c|c|c|c|c|c}
\hline $\mathbf{T}^{\circ} \mathbf{C}$ & $\mathbf{N a}$ & $\mathbf{M g}$ & $\mathbf{A l}$ & $\mathbf{S i}$ & $\mathbf{P}$ & $\mathbf{S}$ & $\mathbf{C l}$ & $\mathbf{C a}$ \\
\hline 1000 & 0,12 & 0,29 & 0,31 & 1,32 & 18,8 & 0,42 & 2,5 & 71,7 \\
\hline 1100 & 0,20 & 0,18 & 0,16 & 1,23 & 18,7 & 0,34 & 2,7 & 70,7 \\
\hline 1200 & 0,23 & 0,37 & 0,36 & 1,26 & 18,3 & 0,52 & 2,3 & 73,2 \\
\hline Hueso [12] & 0,70 & 0,55 & & 0,05 & 11,5 & & 0,1 & 24,5 \\
\hline Hidroxiapatita & & & & & 18,5 & & & 39,6 \\
\hline
\end{tabular}

Con el fin de comparar elementalmente los contenidos de los distintos set de probetas, se obtuvieron datos desde EDS (tabla 2). La estabilidad cristaloquímica del sistema en todo su conjunto, las fases despliegan cambio con una progresiva transformación de las apatitas presentes dentro las más estables formas del fosfato [13, 22-24]. Por otra parte, la desaparición de la sílice cristalina indica la formación de la fase vítrea, figura 4. A pesar de la alta temperatura y alta presión, hay una mayor resistencia a formar alguna fase no cristalina, esto podría atribuirse a la ausencia de fases mineralógicas que actúen como fundente, especialmente algunos feldespatos comúnmente asociados a estas mineralogías. La flogopita aparece a los $1200^{\circ} \mathrm{C}$, pero en una cantidad muy reducida que no fue considerada en el grafico del figura 4. 


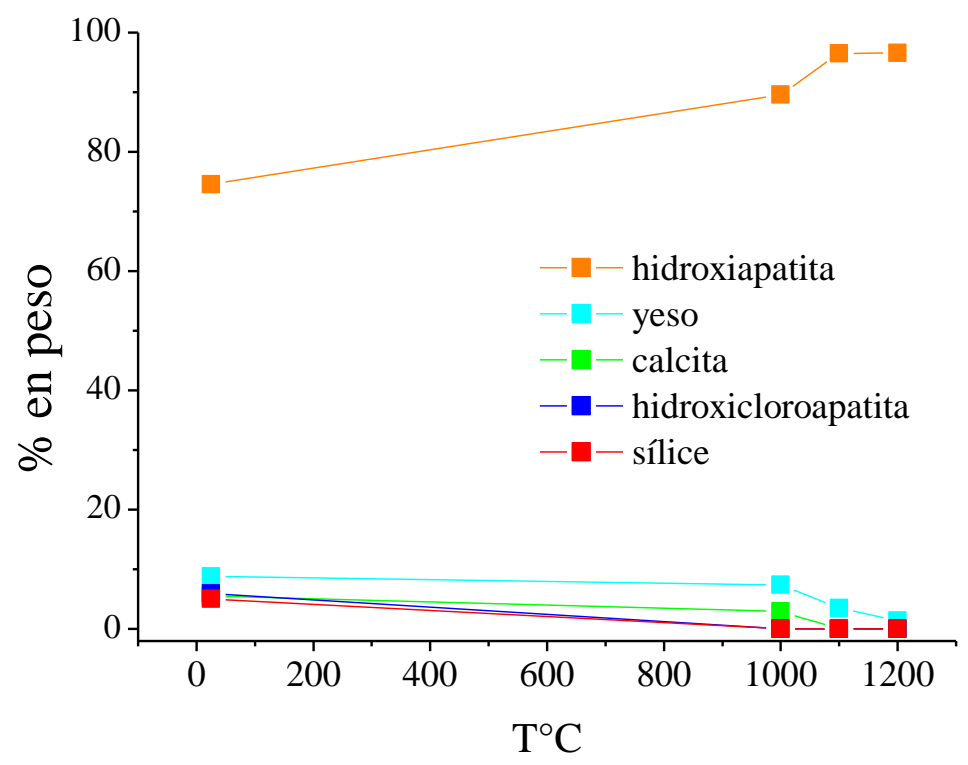

Figura 4: Progreso de las fases con la sinterización.

Se observó, en el tratamiento mecánico de las superficies de las probetas, una buena respuesta a las herramientas abrasivas a medida que aumenta la temperatura de sinterización. De hecho la estabilidad del fosfato en función de la temperatura de sinterización presenta una importante correlación con lo reportado por Elliot [1] $]$.

\subsection{Comportamiento biológico de los sinterizados}

Los test de adhesión y proliferación celular, se realizaron para estimar las primeras interacciones de las células osteoblasticas con la superficie de las muestras sinterizadas. En la prueba de adhesión, el número de células adheridas a la superficie de las probetas se evaluó en bases a la actividad y el área cubierta por estas luego de $48 \mathrm{~h}$ de cultivo. Los resultados del set de probeta S-APAT-1000 muestran un buen grupo celular adheridas.

Sin embargo, las características morfológicas de estas células son una indicación del comienzo de estrés, mostrando una insuficiente extensión celular manifestando una desfavorable vitalidad, lo que origina ausencia de filipodos e/o interconexiones entre ellas, figura 5(a). Una forma cilíndrica del cuerpo de las células, indudablemente indica una deficiente bioactividad sobre las superficies de las probetas [7]. De acuerdo a este punto es evidente entonces, que al aumentar la temperatura de sinterización incrementa la factibilidad de la superficie de albergar mayor cantidad de población celular. De hecho, existe mayor adhesión celular en el set de probetas S-APAT-1200, figura 5(b). 


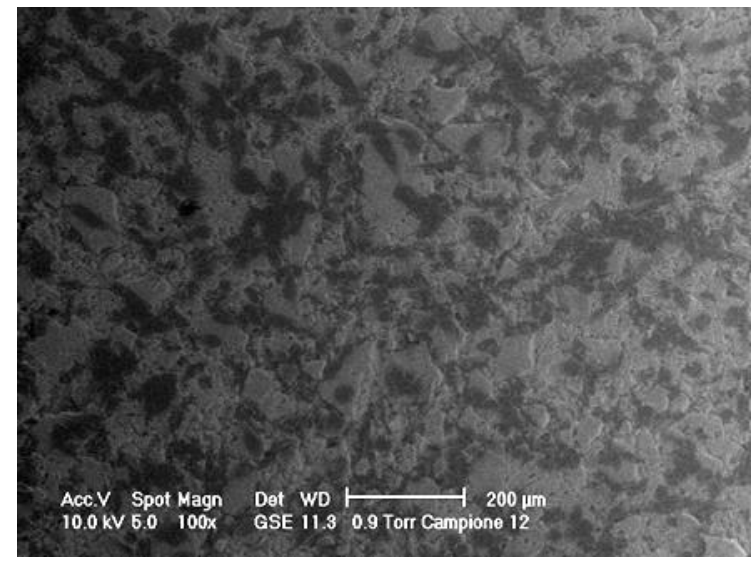

(a)

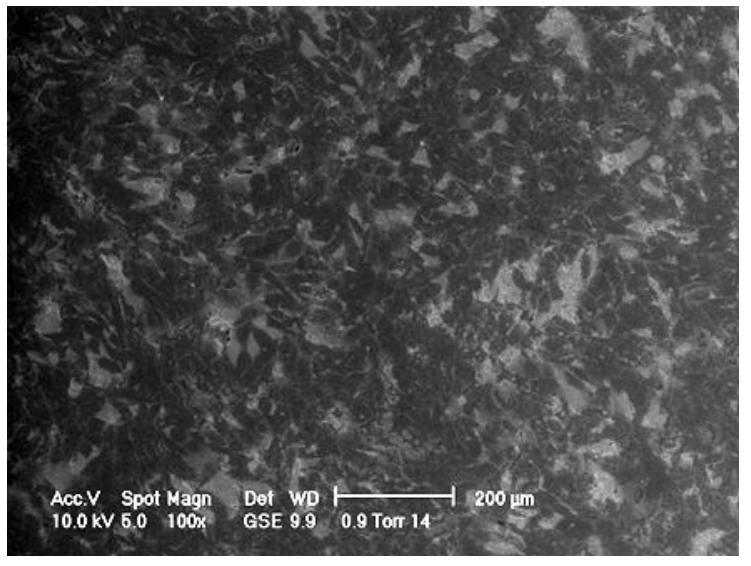

(b)

Figura 5. Test de adhesión y proliferación celular: (a) probeta S-APAT-1000 y (b) probeta S-APAT-1200

Desde el punto de vista de la biocompatibilidad, el set de probetas S-APAT-1200, presentaron un gran incremento de células adheridas con respecto a las probetas producidas a más baja temperatura como se muestra en las figuras 5(a) y 5(b). En este caso, también es posible observar la confluencia de las células en estado funcional y de muy buena vitalidad. Asimismo, las células comienzan a extenderse a dimensiones significativas produciendo buenas interconexiones entre ellas, mostrando una buena bioactividad en la superficie de las probetas de mayor temperatura de sinterización.

\section{CONCLUSIONES}

El depósito de apatita hidrotermal estudiada en este trabajo es una gran fuente de recursos que tiene una interesante concentración de fosfatos, de muy buena homogeneidad y de fácil disponibilidad. La inestabilidad de los átomos de sustitución $\mathrm{F}^{-}, \mathrm{Cl}^{-}, \mathrm{OH}^{-}, \mathrm{CO}^{2-}{ }_{3}$ de la estructura cristalina de la apatita a los procesos térmicos hacen que el material presente buenas condiciones para generar un material no-toxico, pudiendo consolidar componentes densas con adecuadas propiedades físicas y biológicas. La composición del depósito hidrotermal característico del norte de Chile es un modelo adecuado para la reingeniería de materiales biocompatibles. Pese a la ausencia de fases fundentes el material puede consolidarse a altas temperaturas, lo que permite eliminar componentes toxicas en forma de gas. La mejor consolidación de los polvos y el mejor comportamiento biológico se obtuvo en la probeta con más alta temperatura de sinterización, a $1200^{\circ} \mathrm{C}$. Además, es interesante destacar la pureza del material de partida con características similares a los polvos comerciales. Se puede agregar que el material de origen natural hidrotermal tiene una interesante posibilidad de ser un buen candidato para producción masiva de biocomponentes. Pruebas preliminares in vitro indican que el material propuesto muestra buena respuesta a la proliferación y activación de los osteoblastos. Estudios adicionales están en progreso para evaluar la cinética celular y observar la producción molecular en una matriz extracelular junto a su respectiva mineralización. Por tanto, el depocentro hidrotermal tiene buenas posibilidades de ser una solución de bajo costo y altamente viable para la elaboración de componentes para aplicaciones ortopédicas.

\section{AGRADECIMIENTOS}

Se agradece a todos los colegas que hicieron posible este trabajo tanto en la Universitá degli studi di Trento, el apoyo del Instituto de Materiales y la DID de la Universidad Austral de Chile.

\section{BIBLIOGRAFÍA}

[1] STRNAD, Z., SESTAK, J., "Bio-compatible Ceramics as Mimetic Material for Bone Tissue Substitution", In: Proceedings Second International Conference on Intelligent Processing and Manufacturing of Materials, v. 1, p. 151-159, Honolulu, HI(EE:UU.): Plastics Materials, 1999.

[2] HOLZAPFEL, B., REICHERT, J., SCHANTZ, J., et al, "How smart do biomaterials need to be? A translational science and clinical point of view”, Advanced Drug Delivery Reviews, v. 65, n. 4, pp. 581-603, 2013. 
PESENTI, H.; RÍOS, R.; LEONI, M., MOTTA, A., SCARDI, P. revista Matéria, v. 19, n. 3, pp. 247-256, 2014.

[3] SHEPHERD, J., BEST, S., "Calcium Phosphate Scaffolds for Bone Repair", JOM, v. 63, n. 4, pp.83-92, 2011.

[4] DOROZHKIN, S., "Bioceramics of calcium orthophosphates", Biomaterials, v. 31, pp. 1465-1485, 2010.

[5] SAFRONOVA, T., SHEKHIREV, M., PUTLY AEV, V., et al., "Hydroxyapatite-Based Ceramic Materials Prepared Using Solutions of Different Concentrations", Inorganic Materials, v. 43, n. 8, pp. 901-909, 2007.

[6] CLARKE, S., WALSH, P., MAGGS, C., et al., "Designs from the deep: Marine organisms for bone tissue engineering", Biotechnology Advances, v. 29, pp.610-617, 2011.

[7] PESENTI, H., LEONI M., MOTTA, A., et al., "Fossils as Candidate Material for Orthopedic Applications", Journal of Biomaterials Applications, v. 25, n.5, pp. 445-467, 2011.

[8] TAYLOS, B., ANDRIC, T., FREEMAN, J., "Recent Advances in Bone Graft Technologies", Recent Patents on Biomedical Engineering, v. 6, pp. 40-46, 2013.

[9] BEN-NISSAN, B., "Natural bioceramics: from coral to bone and beyond", Current Opinion in Solid State and Materials Science, v. 7, pp. 283-288, 2003.

[10] ROUVILLAIN, J., ROUDIER, M., BOUCHON, C., et al., "Natural Caribbean Coral used for Surgical Implants", The Journal of Bone \& Joint Surgery, v. 77B, pp. 226-227, 1995.

[11] ZHANG, X., VECCHIO, K.S., "Conversion of Natural Marine Skeletons as Scaffolds for Bone Tissue Engineering", Frontiers of Materials Science, pp.1-15, May 2013.

[12] DOROZHKIN, V.D., "Calcium Orthophosphates in Nature, Biology and Medicine”, Materials, v. 2, pp. 399-498, 2009.

[13] ELLIOTT, J.C., Structure and Chemistry of the Apatites and Other Calcium Orthophosphates, Studies in Inorganic Chemistry, v. 18, Elsivier, 1994.

[14] KLEY, J., MONALDI, C.R., SALFITY, J.A., “Along-strike segmentation of the Andean foreland: causes and consequences", Tectonophysics, v. 301, pp.75-94, 1999.

[15] LUCASSEN, F., FOWLER, C.M.R., FRANK, G., "Formation of magmatic crust at the Andean continental margin during early Mesozoic: a geological and thermal model of the North Chilean Coast Range", Tectonophysics, v. 262, n.1-4, pp. 263-279, 1996.

[16] OLIV ARES, V., CEMBRANO, J., ARANCIBIA, G., et al., "Significado tectónico y migración de fluidos hidrotermales en una red de fallas y vetas de un Dúplex de rumbo: un ejemplo del Sistema de Falla de Atacama", Andean Geology, v. 37, n. 2, pp.473-497, 2010.

[17] TRELOAR, P., COLLEY, H., "Variations in $\mathrm{F}$ and $\mathrm{Cl}$ contents in apatites from magnetite-apatite ores in northern Chile, and their ore-genetic implications", Mineralogical Magazine, v. 60, n. 399, pp.285-301, 1996.

[18] TRELOAR, P., COLLEY, H., "Magnetite-Apatite ores in the Atacama fault zone, el Salvador, region, Northern Chile", In: Symposium International Geodynamique Andine, 2nd, pp. 487-490, Oxford, Angleterre, 21-23 Septembre, 1993.

[19] ORRIS, G.J., CHERNOFF, C.B., "Data Set of World Phosphate Mines, Deposits, and OccurrencesPart B. Location and Mineral Economic Data", USGS science for a changing world, Open-File Report, 02156-B, USA, 2002.

[20] DEPARTMENT FOR INTERNATIONAL DEVELOPMENT, "Local phosphate resources for sustainable development in Central and South America", In: Minerals and Geochemical Baseline Programme British geological Survey, Report CR/02/122/N, UK, 2002.

[21] NIKCEVIC, I., JOKANOVIC, V., MITRIC, M., et al., "Mechanochemical synthesis of nanostructured Fluorapatite/fluorhydroxyapatite and carbonated fluorapatite/fluorahydroxyapatite", Journal of Solid State Chemistry, v. 177, pp. 2565-2574, 2004.

[22] MATTHEWS, A., "The decarbonation of carbonate-FAp (francolite)", American Mineralogist, v. 62, pp. 565-573, 1977.

[23] TÕNSUAADU, K., PELD, M., BENDER, V., “Thermal analysis of apatite structure”, Journal of Thermal Analysis and Calorimetry, v.72, pp.363-371, 2003.

[24] LAFON, J.P., CHAMPION, E., BERNACHE, B., et al., "Thermal decomposition of carbonated calcium phosphate Apatites", Journal of Thermal Analysis and Calorimetry, v. 72, n. 3, pp.1127-1134, 2003. 